\title{
ECO-EVOLUTIONARY ORIGINS, NATURE, AND IMPACT OF PAIRED REPRODUCTION IN EARTH AND POSSIBLE EXTRATERRESTIAL MICROBIOTA
}

Kevin B. Clark, Ph.D.

(kbclarkphd@yahoo.com and kevin.clark@mpg-alumni.de)

Director, Felidae Conservation Fund, Mill Valley, CA 94941, USA; Co-Chairperson, Science Advisory Board, Cures Within Reach, Chicago, IL 60602, USA; Domain Champion in Biomedicine and Campus Champion, NSF Extreme Science and Engineering Discovery Environment (XSEDE), National Center for Supercomputing Applications, University of Illinois at Urbana-Champaign, Urbana, IL 61801, USA; Affiliate, NASA Ames Research Center, Mountain View, CA 94035, USA; Affiliate, NASA NfoLD, NASA Astrobiology Program, NASA Ames Research Center, Mountain View, CA 94035, USA; Advisor, Universities Space Research Association, Columbia, MD 21046, USA; Subject Matter Expert and Mentor, Penn Center for Innovation, University of Pennsylvania, Philadelphia, PA 19104, USA; Consultant and Collaborator, Research and Development Service, Veterans Affairs Greater Los Angeles Healthcare System, Los Angeles, CA 90073, USA; Main Organizer, Shared Interest Group for Natural and Artificial Intelligence (sigNAI), Max Planck Alumni Association, 14057 Berlin, Germany; Member, Nanotechnology and Biometrics Councils, Institute for Electrical and Electronics Engineers (IEEE), New York, NY 10016-5997, USA.

White Paper Running Head: ClarkKevinB1, Eco-Evolution of Microbial Paired Reproduction 


\begin{abstract}
Major Earth taxa, including microbiota, evolved sex-like mechanisms, limiting genomic fixation of harmful mutations while enabling genetic innovations to strengthen cohort and progeny selective advantages, But, imperfect scientific descriptions leave the origins, nature, and impact of sex on Earth and perhaps other livable worlds poorly understood.
\end{abstract}

\title{
BACKGROUND
}

Sex, weakly defined as the exchange and recombination or reassortment of genetic material during paired reproduction, helps keep harmful mutations from becoming fixed within genomes of isolated populations while perhaps enabling genetic innovations that strengthen selective advantages for progeny and, in instances of horizontal gene transfer of vertically evolved genes, cohorts. Viruses, bacteria, fungi and molds, protozoa, plants, and animals all have evolved sexual or sex-like mechanisms, with increasingly sophisticated eukaryotic cellular life generally depending on isogametes, anisogametes, or oogametes to deploy genomes $(1,5,6,8,9,13-15)$. Mate differentiation, such as different sexes or mating types defined by di- or polymorphisms and other features, represents significant evolutionary, ecological, and developmental sociobiological transitions in life on Earth that may lead to further transitions and, though often ignored by most astrobiologists, may also accompany and drive possible life found elsewhere in the universe, such as suitable ocean worlds with biospheres capable of supporting cosmopolitan microbiota through bioenergizing rock-water serpitinization reactions or solar and/or planetary geothermal based nutrition production. Yet prevailing opinions insist only animals co-evolved the somatic structures (i.e., ornaments and weapons), behavioral routines (e.g., courtship and dominance rituals), and decision making processes (e.g., learning, memory, and deduction) needed for the sorts of complex sexual selection first described by Darwin and later by founders of the neoDarwinian school of thought $(1,5,6,8,12)$. Darwinian and neoDarwinian frameworks of sexual selection may be categorically broken into intersexual and intrasexual mechanisms through which social judgments about mate availability, quality or competence, and compatibility can be inferred by courting couples and by rivals. Intersexual selection serves to motivate and coordinate the mating activities of partners in space and time. In contrast, intrasexual selection serves to discourage or prevent successful sexual relations of rival suitors or recipients contending for the same prospective mate(s).

Phycologists, bacteriologists, and protistologists now recognize that aspects of unicellular microorganism morphology and behavior more-or-less anecdotally reported by early twentiethcentury scientists fulfill at least some criteria of mating ornaments and courtship. Enterobacteria, algae, yeast, cellular slime molds, and ciliated protozoa, for example, secrete diffusible peptide mating pheromones that motivate intraspecies and sometimes interspecies mates to engage them in either sexual-like monogamous conjugation or other forms of paired reproduction $(1,5,6,8,12)$. When detected, gradients of circulating mating pheromones advertise the location and sex or type of mate, automatically inducing an instinctual-like formation of sticky proteins and a conjugation bridge and/or other changes in cell structure with which either both cells fuse and exchange genes or a "male" donor cell physically docks then initiates gene transfer to a "female" recipient cell. For nonmotile microbes, such as some algae, enterococcal bacteria, and fungi, pheromone-triggered intraspecies mate selection is a largely random outcome of dynamic ecological conditions favoring cell proximity and contact. But microbes competent at $\mathrm{Ca}^{2+}$ -

White Paper for Decadal Survey on Planetary Science and Astrobiology Page 2 of 8 
dependent social twitching, flagellar propulsion, ciliary reversals, and additional forms of locomotion amounting to courting dances and dominance displays figure to be skilled at selecting compatible fit mates and at deterring rivals, thus gating the spread of inferior genes due to promiscuous unions. Indeed, such behavior is governed by Hebbian-like learned social logics in ciliated protozoa capable of deceptively or altruistically signaling mating availability and prowess. In particular, contractile ciliates, unlike most other microbes, have been shown to plan, search, and execute learned social strategies, such as switching messages of behavioral propaganda and putatively bestowing beneficial epigenetic "nuptial gifts", in an effort to minimize survival/reproductive tradeoffs $(1-8,10,12)$. Mating decisions reached by these microbes resemble learned social inferences derived from availability and representativeness heuristics of suitor/recipient fitness and correspond to the decision making processes used by "higher" animals experiencing true sexual contexts. Such phenomena provide powerful mechanisms for microbes to adapt to and exploit their environs, enabling niche creation and dominance in previously inhospitable to hospitable eco-evolutionary settings.

\section{MICROBIAL MATE DIFFERENTIATION AND SELECTION}

Prior emphasis on trying to understand the origins and purposes of animal sexual behavior had revealed that both genetically predisposed instincts and learned behavioral plasticity contributed to the evolution of species and to adaptability of an individual organism within its lifespan. The same kind of approach with microbes has led to similar conclusions, profoundly changing our concepts about the complexity of microbial social interactions and gene transfer leading to adaptations, such as tolerance to stressors and toxins, pathogen virulence, biofilm formation, and cell aggregation (1-15). Contrary to popular, largely unsubstantiated beliefs held by scientists studying animal perisexual behavior, many microbes, ranging from bacteria to algae to protozoa, present rudimentary aspects of mate selection during intact microbe, gamete, or spore stages of lifecycles. Acts of intra- and intermate selection by microbes may incorporate, among other events: secretion of mating pheromones that announce potential partner location and reproductive compatibility and that initiate changes in cell structure and function needed for cell docking and gene transfer, anatomical and behavioral di- or polymorphisms which covary with reproductive success and which respectively differentiate sexes and mating types, pheromoneelicited stereotypic behaviors that serve to motivate, orient, and coordinate attracted compatible partners into reproductive unions, and/or learned heuristic-guided social logics used to reciprocate with presumptive rivals and suitors in an effort to gain reproductive advantages (18,10-14).

The above and other accumulating research findings establish that microbes are capable of both instinctual-like and learned mating behaviors previously only attributed to animals. Genetically inherited characteristics, such as life history traits, certain cheater genotypes, mechanisms and structures for cell-cell attachment, intercellular signaling, and motility apparati, provide the basis for cooperative and competitive social behaviors exploited by microbes in reproductive contexts (1-8,10-15). Epigenetic modifications in phenotype, such as environmentally induced changes in motility competence and cell morphology, and traditional forms of dual-process nonassociative and associative learning and memory, best documented in eukaryotic protozoa, tune genetic behavioral predispositions to facilitate joint fitness and reproductive success between mating partners. Although fungi and algae have evolved true sex, the most sophisticated microbial mating practices currently known to scientists are those expressed by ciliated protozoa. 
Heterotrich ciliates, for instance, are capable of complex ritualistic courtship dances and dominance displays, of drawing representativeness or probability inferences about the survival and reproductive fitness of conspecifics, of exchanging epigenetic "nuptial gifts" that restore motility and increase paired fitness during conjugation, and of deceptively and altruistically signaling mating availability and prowess (1-6,8,10,12). Given these sorts of perimating strategies are mediated by learned heuristics and primitive social logics, other behaviors planned and executed by animals to find, choose, and obtain mates are possibly within the behavioral repertoires of ciliates and perhaps more primitive microbes.

\section{MICROBIAL MATING ORNAMENTS, WEAPONS, BEHAVIORAL ROUTINES, AND DECISIONS}

True sex by microbes, particularly fungi and green algae, was well known to microbiologists of the late nineteenth and early twenty centuries. Today, arguably the best models of sexual reproduction in microbes are the prevalent ascomycetous and the less abundant basidiomycetous yeasts. Laboratory studies involving these fungi have caused recent explosions in data detailing subtle variations in cell structure and function that might form bases for mate selection. The proper or true fungi, composed of phycomycetes, basidiomycetes, and ascomycetes, such as Saccharomyces cerevisiae, maintain complete sexual cycles with maturating sexual structures, including gamete-filled gametangium and "shmoo" formations (i.e., a pear-shaped cellular geometry with apical mating projection or conjugal bridge for nuclear transport), while imperfect fungi only proliferate asexually (8). Some formerly classified imperfect fungi, such as strains of ascomycete Candida albicans, show infrequent sexual activity stemming from cryptic mating alleles and machinery. Dimorphisms in a single gene locus, called the mating type locus, underlie bipolar mating systems for homothallic and heterothallic populations of ascomycete, basidiomycete, and zygomycete phyla (8). Many basidiomycete yeast also use a tetrapolar mating system comprised of two unlinked sex-determining gene loci for homothallic and heterothallic reproductive unions. Mating type loci encode for specializations that express sex or mate-type identity, heterodimer transcription factors, mating pheromones, and plasma-membrane G-protein-coupled pheromone receptors and a mitogen-activated protein kinase pathway that transduce mating signals into gene transcription required for cell-cycle arrest, cytoskeletal rearrangement, cell polarization or orientation toward the compatible pheromone source, cell fusion, membrane degradation, and nuclear migration and fusion (8).

Depending on the mating system, two to thousands of fungal mating types may be formed. Interestingly, polymorphic mating systems are believed to be maintained by negative frequencydependent selection. That is, when a mating-type allele becomes rare, its selective advantage increases since microbes carrying the allele can mate with a larger proportion of nonself individuals (8). Hence, morphological characteristics secondary to gamete copulation which covary with mating allele frequency should also act as propaganda, either as anatomical weapons or ornaments, of viability and reproductive success. Such a hypothesis awaits confirmation for various characteristics. However, phenotypic changes in cell pigmentation, from white to opaque (or opaque to white), occur during the transition between asexual and sexual competence in Candida albicans and might be an ornamental precursor signifying potential reproductive success to prospective mates and rivals. Moreover, evidence for qualitative differences in expression of adhesion proteins required for cell docking, detection accuracy, sensitivity, and 
error-correction in pheromone recognition, and rate and size of shmoo formation and conjugal bridge growth likely grant selective advantages when competing with rivals during intramate selection and when pursuing mates during intermate selection (8).

In addition to fungi, bacteria, cellular slime molds, single-celled and filamentous green algae, and protozoa reasonably possess anatomical and physiological di- or polymorphisms capable of being used as weapons and/or ornaments during mate selection. The exact facts of cell physiology and anatomy for other microbes differ (8). But since many of the central concepts of cell differentiation, such as mating type, pheromone synthesis, secretion, and detection, and cellcell docking useful for understanding fungal mate selection and gene exchange, can be applied to the sexual or sexual-like practices of other eukaryotes, a specific discussion of cellular slime mold, green algae, and protozoa mating weapons and ornaments will be eschewed for brevity. Rather, the white paper now focuses on contrasting and comparing key mating traits of prokaryotes with those of eukaryotes. Bacteria can transfer collections of genes, termed plasmids, or gene fragments to other bacteria of the same or different species and genus and to members of protist, plant, and animal taxa (8). Unlike in eukaryotes, this process is meiotically independent and involves typical unidirectional passage of genes, via a conjugation bridge or pilus, which become incorporated into the genome of the recipient cell. Some bacteria are more successful than others at conjugation, a process favorable to improved viability, such as procuring antibiotic resistance genes. For example, the gastrointestinal Gram-positive pathogen, Enterococcus faecalis, engages in bacterial conjugation and horizontal gene transfer at greater frequencies because of selective expression and secretion of short peptide mating pheromones (8). One of the best studied pheromones, cCF10, is encoded by the genes of recipient cells lacking the plasmid pCF10. After proteolytic hydrolysis of its precursor protein, the pheromone is released into the extracellular environment to attract donor cells harboring the pCF10 plasmid. Like pheromone-dependent reproduction in nonmotile fungi, nonmotile Enterococcus faecalis recipients must wait until suitable nonmotile donor cells come in close physical proximity, due to random ecological conditions or directed growth, before pheromone-stimulated conjugal bridge extension, conjugation, and transfer of genetic material may begin (8). Activated donors internally assimilate the pheromone and upregulate the expression of adhesin Asc10 and other gene products. The Asc10 molecule extends from the donor cell surface, facilitating contact via the conjugal pilus and, therefore, conjugation between donor and recipient cells. Although mating pheromones can increase "clumping" between rivaling donors, two plasmid-encoded polypeptides, one surface molecule that degrades or sequesters the pheromone and one that inhibits the pheromone receptor, obstruct autocrine signaling and self-induced conjugativeplasmid transcription (8).

Deviations between donors in the geometry of their conjugal bridges and the concentrations of Asc10 produced should yield mating advantages sought by recipient cells to increase the success of gene transfer (8). If these deviations covary with and indeed represent improved gene transfer, they could be considered primitive mating ornaments loosely analogous to, for example, long elaborate avian tail feathers denoting health and ecological fitness or dexterous limbs adapted for gentle caresses and social grooming. Moreover, donor cells capable of degrading or sequestering higher concentrations of pheromones could disrupt with weapon-like precision the conjugal processes of rivals. Conjugal bridges may also serve as horn or lance, ramming rivals away from possible mates in a joust of suitor donor cells. Such putative intra- and intermate selection bring 
into question the belief that bacteria are strictly promiscuous in their choice of mating partners and would add to other phenomena, including obstacles to plasmid entry, resistance to integration and functional incompatibility of nucleotide segments, and recombination reversal by mismatch repair, that control the restriction of interspecies and even interkingdom bacterial conjugation and genome recombination (8).

For animals, it is not enough to have vestigial weapons and ornaments; those adaptations must be used to capture some amount of reproductive advantage. Expertly executed behaviors and decisions accomplish this ambition. Animal mating behaviors often involve, among other phenomenon, locomotor activity to display intricate courtship dances and to aggress against rivals, instances of ostensive and nonostensive communications to proclaim interest in a candidate mating partner, and the giving of a gift or self-sacrifice to establish prowess or emotional intimacy (8). These factors encompass motivational (e.g., social facilitation or enhancement, incentive motivation, etc.) and perceptual influences (e.g., local and stimulus enhancement) of simple and complex social learning, such as discriminated following, observation conditioning, emulation, and imitation. Microbes in many respects are no different than their metazoan descendants. Prokaryotes and "lower" eukaryotes communicate mating signals with pheromones that, in a Darwinian way, attract, orient, and motivate mating partners. Pheromones transmit information about the type and location of mates available as well as the potential fitness of the pheromone source through chemical gradients, concentrations, and release durations. Attracted cells automatically and preferentially grow, swim, crawl, or glide toward pheromone sources perceived as being most fit, such as yeast cells or ciliated protozoa that secrete higher concentrations of pheromones. High pheromone concentrations released and differentially detected by microbes for the purpose of cell-cell communication and mate discrimination are thus analogous to elaborate language play by humans during courtship situations.

This sort of ritualized ornamental behavior in human culture connotes levels of mental acuity and health in addition to other parameters indexing survival and reproductive fitness. Skilled communication by microbes also might expose levels of viability. However, the decision to select one potential mate over another is not simply determined from chemical signals. For example, preconjugal ciliates, such as the hypotrichs Oxytricha bifaria and Euplotes rakovi, iteratively exhibit a combination of exploring and avoidance reactions consisting of jerking motions, back- and sidesteps, and pivots in response to mating pheromones $(1,2,4,5,8)$. These $\mathrm{Ca}^{2+}$-dependent stereotypic maneuvers, showing fair resemblance to animal courtship displays, are coordinated between presumptive mating partners of nonself mating types into a courtship dance which helps spatially orient microbes and induce cell-cell touches and changes in membrane structure and excitability leading to cell fusion and paired reproduction. Completed conjugation in ciliates, dissimilar to bacteria, is a terminal event in the lifecycle of parent cells, so this mating practice, if successful, could be considered a highly selective monogamous event conferring selected traits onto daughter cells. Complex mating dances in motile bacteria remain unreported in the peer-reviewed literature, but motile species of cyanobacteria, enterococci, and additional bacteria might possess that capacity (8). For example, Streptococcus sanguinis strains competent at probable $\mathrm{Ca}^{2+}$-dependent "social" twitching figure to be skilled at courting compatible fit recipients and at deterring donor rivals prior to delivery of transposons (e.g., Tn919) to fellow bacteria. Depending on the number of successful unions achieved by a 
bacterium, such selective horizontal gene transfer might be considered a polygamous mating strategy, perhaps gaiting the invasion of beneficial genes into inferior populations of, for instance, motility-incompetent bacteria. In general, the quality and fidelity of mating communications and choices made by prokaryotic or eukaryotic microbes generates tremendous ecological advantages for the mated pair and resulting progeny, such as tolerance to stressors and toxins, pathogen virulence, biofilm formation, and cell aggregation that shape life conditions for both ambient and host niches (1-15).

\section{CONCLUSIONS}

Despite almost two centuries of the experimental and theoretical examination of sex and alternative reproductive mechanisms with modern scientific methods, little remains known about the origins and evolution of these processes on Earth and possibly elsewhere in the universe (8). For example, assortative pairings between reproductively mature organisms are believed to increase (i.e., positive assortative pairings) or decrease (i.e., negative assortative pairings) trait variation through mechanisms of mate selection and monogamy or polygamy. Darwin and later neoDarwinists largely regarded these mating processes as a matter of supply and demand economics, where mismatched competitors, as separate individuals or groups of individuals, vie for and acquire or lose limited preferred inter- and intrasexual resources and, therefore, maximize speciation by minimizing costly evolutionary tradeoffs which endanger fertility, reproductive success, and survival (8). Regardless of whether humans, animals, or phylogenetically older life forms evolved mating practices, the ability to make inferences or judgments about the survival and reproductive fitness of prospective mating partners confers probabilistic, if sometimes suboptimal, selection decisions favoring overall passage of ecologically salient characteristics to future generations. But these events are tremendously complicated and many models, principles, and theories extending Darwin's original ideas, including Bateman's principle, Fisher's Geometric Model of Adaptation, the Handicap principle, Muller's Theory of Dominance, Sexual Conflict and the Evolutionary Arms Race, NK Models, and Extreme Value Theory, have been unsuccessfully conceived or modified to account for environmental pressures driving special cases of mate selection and trait frequencies within populations diverging from conventional Darwinian evolution (8).

Broadening axiomatic limits of older models, principles, and theories or to create new ones will provide unprecedented descriptive and predictive insights into how and why instincts and learning within and across taxonomic boundaries of single to multicellular life interact with natural selection and promote both competitive and curious noncompetitive phenotypes and behaviors that may possess dubious direct survival or reproductive value, such as maternal infanticide, bestowing nuptial gifts, brood nurturing by uncles and aunts, homosexuality, deceptive and honest mating propaganda, perception and covetousness of physical beauty, excessively creative oral and written language use, and cuckoldry and female promiscuity (8). Challenges to the standard doctrine of sex and mate selection will moreover help identify, characterize, and explain types of mate selection employed by microbes in a variety of ecoevolutionary scenarios that favor or do not favor sexual or sex-like reproduction compared to other forms of microbial reproduction (e.g. binary fission). Future studies should also seek to determine if microbes might perform various primitive analogues of multicellular organism mating behaviors, such as mate copying, nonostensive or concealed communications to induce and consummate mating relations where eavesdropping by rivals may threaten conjugal success, 
mimicry of the opposite sex or mating type (e.g., female mimicry and transsexual behavior) to coerce rivals away from prospective mates, and "allegiant" cooperation with kin of courted partners to guarantee reproductive goals (1-15),. Such efforts will help contextualize the role of paired reproduction in major eco-evolutionary transitions evident on Earth and perhaps provide scientific understanding and (gnostic and agnostic) biosignatures of similar processes within extinct and extant biospheres throughout the universe.

\section{REFERENCES}

1) Clark, K.B. (2010). Origins of learned reciprocity in solitary ciliates searching grouped 'courting' assurances at quantum efficiencies. BioSystems, 99(1), 27-41.

2) Clark, K.B. (2010). Bose-Einstein condensates form in heuristics learned by ciliates deciding to signal 'social' commitments. BioSystems, 99(3), 167-178.

3) Clark, K.B. (2010). On classical and quantum error-correction in ciliate mate selection. Communicative \& Integrative Biology, 3(4), 374-378.

4) Clark, K.B. (2010). Arrhenius-kinetics evidence for quantum tunneling in microbial "social” decision rates. Communicative \& Integrative Biology, 3(6), 540-544.

5) Clark, K.B. (2012). Social biases determine spatiotemporal sparseness of ciliate mating heuristics. Communicative \& Integrative Biology, 5(1), 3-11.

6) Clark, K.B. (2013). Ciliates learn to diagnose and correct classical error syndromes in mating strategies. Frontiers in Microbiology, 4, 229.

7) Clark, K.B. (2013). Biotic activity of $\mathrm{Ca}^{2+}$-modulating nontraditional antimicrobial and -viral agents. Frontiers in Microbiology, 4, 381.

8) Clark, K.B. (2013). The mating judgments of microbes. In K.B. Clark (Ed.), Social learning theory: Phylogenetic considerations across animal, plant, and microbial taxa, pp. 173-200. Hauppauge: Nova Science Publishers, Inc. ISBN 978-1-62618-268-4.

9) Clark, K.B. and Eisenstein, E.M. (2013). Targeting host store-operated $\mathrm{Ca}^{2+}$ release to attenuate viral infections. Current Topics in Medicinal Chemistry, 13(16), 1916-1932.

10) Clark, K.B. (2014). Evolution of affective and linguistic disambiguation under social eavesdropping pressures. Behavioral and Brain Sciences, 37(6), 551-552.

11) Clark, K.B. (2014). Basis for a neuronal version of Grover's quantum algorithm. Frontiers in Molecular Neuroscience, 7, 29.

12) Clark, K.B. (2015). Insight and analysis problem solving in microbes to machines. Progress in Biophysics and Molecular Biology, 119, 183-193.

13) Clark, K.B. (2018). Searching for (Proto)Cellular Logics Proteins in Earth-like Environments Hospitable and Inhospitable to Life. White paper submitted to the Committee on an Astrobiology Science Strategy for the Search for Life in the Universe, National Research Council, Washington, D.C.

14) Clark, K.B. (2019). Unpredictable homeodynamic and ambient constraints on irrational decision making of aneural and neural foragers. Behavioral and Brain Sciences, 42, e40.

15) Clark, K.B. (2019). Neurotropic enteroviruses coopt "fair-weather-friend" commensal gut microbiota to drive host infection and CNS disturbances. Behavioral and Brain Sciences, 42, e68. 